\title{
Genome-Wide Association Study (GWAS) For Cold Tolerance at The Bud Burst Stage in Rice Using SNP Markers
}

\section{Caijing Li}

Jiangxi Agricultural University

Jindong Liu

Agricultural Genomics Institute at Shenzhen

Jianxin Bian

Peking University Institute of Advanced Agricultural Sciences

Tao Jin

Jiangxi Agricultural University

\section{Baoli Zou}

Jiangxi Agricultural University

Shilei Liu

Jiangxi Agricultural University

Xiangyu Zhang

Jiangxi Agricultural University

\section{Peng Wang}

Jiangxi Agricultural University

Jingai Tan

Jiangxi Agricultural University

Guangliang Wu

Jiangxi Agricultural University

\section{Qin Chen}

Jiangxi Agricultural University

\section{Yanning Wang}

Jiangxi Agricultural University

Qi Zhong

Jiangxi Agricultural University

Shiying Huang

Jiangxi Agricultural University

Mengmeng Yang

Jiangxi Agricultural University

Tao Huang 
Jiangxi Agricultural University

\section{Haohua He}

Jiangxi Agricultural University

Jianmin Bian ( $\sim$ jmbian81@126.com )

Jiangxi Agricultural University

\section{Research Article}

Keywords: cold tolerance, rice landraces, GWAS, seedling survival rate (SSR), QTL

Posted Date: March 18th, 2021

DOI: https://doi.org/10.21203/rs.3.rs-311085/v1

License: (9) This work is licensed under a Creative Commons Attribution 4.0 International License. Read Full License 


\section{Abstract}

Background: Rice is a crop that is very sensitive to low temperature, and its morphological development and production are greatly affected by low temperature. Therefore, understanding the genetic basis of cold tolerance in rice is of great significance for mining favorable genes and cultivating excellent rice varieties. However, there were limited studies focusing on cold tolerance at the bud burst stage, therefore, considerable attention should be paid to the genetic basis of cold tolerance at the bud burst stage (CTBB).

Results: In this study, a natural population consisting of 211 rice landraces collected from 15 provinces of China and other countries were firstly used to evaluate the cold tolerance at the bud burst stage. Population structure analysis showed that this population divided into three groups and was rich in genetic diversity. Our evaluation results confered that the japonica rice was more tolerance to cold at the bud burst stage than indica rice. Genome-wide association study (GWAS) were performed through the phenotypic data of 211 rice landraces and 36,727 SNPs dataset under a mixed linear model, and 12 QTLs $(\mathrm{P}<0.0001)$ were identified according to the seedling survival rate (SSR) treated at $4{ }^{\circ} \mathrm{C}$, in which there are five QTLs ( $q S S R 2-2$, qSSR3-1, qSSR3-2, qSSR3-3 and $q S S R 9$ ) which were co-located with previous studies, and seven QTLs (qSSR2-1, qSSR3-4, qSSR3-5, qSSR3-6, qSSR3-7, qSSR4 and qSSR7) which were reported for the first time. Among these QTLs, qSSR9, harboring the highest-peak SNP, explained biggest phenotypic variation. Through bioinformatics analysis, five genes (LOC_Os09g12440, LOC_Os09g12470, LOC_Os09g12520, LOC_Os09g12580 and LOC_Os09g12720) were nominated as candidates for qSSR9.

Conclusion: This natural population consisting of 211 rice landraces with high density SNPs will serve as a better choice for identifying rice QTLs/genes in future, and the detected QTLs associated with cold tolerance in rice bud burst stage will be conducive to further mining favorable genes and breeding of rice varieties under cold stress.

\section{Background}

Originated in the tropical and subtropical, rice is one of the main staple foods in the world. Low temperature has a huge impact on rice. More than 15 million hectares of rice cultivation area in the world have been persecuted by low temperature. Severe cold damage exists in many countries, mainly in Japan, South Korea, the United States and China [1]. Rice is cultivated in a wide area in China, ranging from $53^{\circ}$ 27 'to $18^{\circ} 90^{\prime}$ north latitude, especially in the provinces of the Yangtze river basin in China, from 1951 to 1980, japonica rice and indica rice in the Yangtze river suffered from cold injury severely, the disaster areas lose 5 to 10 billion $\mathrm{kg}$ of rice every year [2]. Early rice in the Yangtze river basin in China is often affected by cold injury at the bud burst stage, resulting in low germination rate and failure to emerge. Therefore, it is very necessary to study the cold tolerance in rice at the bud burst stage.

Cold tolerance is a complex quantitative trait that is often controlled by multiple genes and environment, and researchers often use bi-parental populations to look for QTLs associated with cold tolerance. The 
researchers excavated more than 250 QTLs in the bi-parental population using traditional QTL mapping methods during various stages of rice growth [3]. Among more than 250 QTLs, many genes throughout the rice whole growth stages have been isolated. At the germination stage, qLTG3-1 was the first gene to be linked to germination at low temperatures [4]. During the seedling stage of rice, many QTLs/genes related to cold tolerance of rice were isolated, including qCTS12 [5], qCTS4 [6], qCtss 11 [7], qSCT1 and qSCT11 [8], qLOP2 and qPSR2-1 [9], COLD1 [10] and qCTS-9 [11]. qCTS12 was the first identified coldtolerance gene at seedling stage. COLD1 is another important gene related to cold tolerance in rice seedling stage, and it is also the first cold-tolerance gene involved in signal transduction. At the booting stage of rice, several genes have been isolated, including Ctb1 [12], qCT8 [13], qCTB7 [14], qCTB3 [15] and $q C T-3-2$ [16], Ctb1 is the first gene to be linked to cold tolerance at booting stage in rice. Although biparental mapping populations have played a great role in traditional gene mapping, the construction of bi-parental populations entails a major investment in time, and has therefore limited the number of genes excavate to date [17].

In order to excavate more new genes associated with target traits, the exploration of QTLs/genes through the natural population with weak genetic relationship using GWAS has become one of the most popular methods. This method eliminates the need to construct a mapping population, and can simultaneously analyze multiple alleles, using the recombination information from the long-term evolution of natural populations. In recent years, some studies have applied this method to study rice cold tolerance. 17 QTLs were detected related to the rice germination at low temperatures using 63 Japanese varieties [18]. 51 QTLs were mapped by GWAS with the population of 174 rice accessions from China [19]. 132 QTLs were identified from 527 rice varieties for both rice natural chilling and cold shock stresses [20]. 67 QTLs were mapped for cold stress at the seedling stage of rice, 56 QTLs were newly discovered [21]. 42 QTLs were found associated with cold tolerance in rice seedling stage, 20 of which have not been mentioned in previous reports [22]. 31 QTLs were detected related to low temperature germination of rice seeds at rice germination stage using 200 japonica rice varieties [23]. 47 QTLs were identified for cold tolerance at the bud burst stage using 249 indica rice accessions [24]. 26 QTLs were found related to cold tolerance in rice seedling stage by using a core collection of landraces of rice from 2,262 accessions of Ting's collection [25]. In addition, 31 distinct QTLs regions were identified in a panel of 257 rice accessions from all of the world for low temperature germination [26]. However, there are still few studies using GWAS to explore cold tolerance in rice at the bud burst stage. In order to understand the genetic mechanism of cold tolerance in rice at early stage, it is necessary to search for QTLs related to cold tolerance in rice at the bud burst stage.

In this study, we selected 211 rice landraces from different regions to form a natural population and performed high-throughput sequencing using microarray. The 211 rice landraces were mainly composed of indica and japonica rice, which provided abundant genetic diversity for studying cold tolerance of rice. We treated the natural population with low temperature at $4{ }^{\circ} \mathrm{C}$, and then recovered it at room temperature. A total of 12 QTLs and 5 candidate genes for $q S S R 9$ related to cold tolerance were identified by genome-wide association study of seedling survival rate (SSR), which provided valuable gene 
resources for cold tolerance research in rice and laid a solid foundation for breeding cold tolerant rice varieties.

\section{Results}

\section{Cold tolerance of the 211 rice varieties}

In our study, SSR were used for evaluate the CTBB (Table S2). Due to the abundant landrace germplasm resources, the phenotypes of rice varieties within the natural population varied greatly after $4^{\circ} \mathrm{C}$ treatment (Table 1;Fig. 1). We classify cold tolerance into five levels of SSR: Extremely sensitive $(0 \leq X \leq 20)$, Sensitive $(20<X \leq 40)$, Light sensitive $(40<X \leq 60)$, Tolerance $(60<X \leq 80)$, Extremely tolerance $(80<X \leq 100)$. Of the 101 rice varieties that were extremely sensitive to low temperature, 99 were indica and 2 were japonica. Correspondingly, Of the 69 rice varieties that were extremely tolerance to low temperature, 5 were indica and 64 were japonica. According to SPSS software 26.0 analysis results, the SSR was significantly correlated with indica and japonica, and the correlation coefficient was 0.851 (Table 2), suggesting that japonica was more cold tolerance than indica at the bud burst stage. On the other hand, we found that the cold tolerance of rice was significantly related to its geographical distribution. and the correlation coefficient was 0.714 (Table 2). The higher the latitude, the stronger the cold tolerance of rice, which may be due to the significant correlation between indica or japonica types with latitude (Table 2).

\section{Population structure and relative kinship}

Based on the 36,727 SNPs, the STRUCTURE, Neighbor-joining (NJ) tree method, Principal Component Analysis (PCA) and Kinship were used to analyze the population structure of the natural population (Fig. 2). According to the STRUCTURE analysis, the log likelihood increased gradually from $K=1$ to $K=10$. The maximum ad hoc measure $1 \mathrm{~K}$ was observed for $\mathrm{K}=3$, which indicated that the entire population could be divided into three subgroups (Fig. 2A). The 211 rice varieties could be divided into 3 subgroups by NJ tree (Fig. 2B) and three principal components from this panel (Fig. 2C). In addition, in the relationship analysis, we found that there were two major groups of subgroups and a middle subgroup in the 211 rice varieties (Fig. 2D), suggesting that the landraces population germplasm resources were abundant, which was beneficial for performing GWAS.

\section{GWAS analysis for CTBB}

Based on genotype and phenotype data, association analysis was performed under a mixed linear model using PCA and KINSHIP as co-variables. A total of 12 QTLs (qSSR2-1, qSSR2-2, qSSR3-1, qSSR3-2, qSSR33, qSSR3-4, qSSR3-5, qSSR3-6, qSSR3-7, qSSR4, qSSR7 and qSSR9) were identified in SSR with well-fitted quantile-quantile ( $Q-Q)$ plots ( $p<0.0001$ ) (Fig. 3 and Fig. 4; Table 3). A QTL, qSSR2-2, on chromosome 2 overlapped with OSWRKY71 [28]. Another QTL on chromosome 2, qSSR2-1, has not been previously reported. On chromosome 3, there were three QTLs overlapping with previous studies [29-31], namely qSSR3-1, qSSR3-2 and qSSR3-3, the other four QTLs on chromosome 3 are newly discovered and have not 
been reported by previous studies. The other two QTLs on chromosome 4 ( $q S S R 4$ ) and 7 ( $q S S R$ ) were also newly discovered in this study. In addition, we also found a QTL on chromosome 9, qSSR9, which harbored the highest-peak SNP, which explained biggest phenotypic variation. It is located around 7.1 Mb on chromosome 9, and this QTL co-located with c/r9 [32] .

\section{Candidate gene analysis}

Among these QTLs, we conducted further candidate genetic analysis of the qSSR9. According to the LD decay analysis, a total $244-\mathrm{kb}$ region was identified as the candidate region (Fig. 5). There are 39 genes in this region, including 3 hypothetical proteins, 4 transposon proteins, 7 retrotransposon proteins and 16 functionally annotated genes (Table S3). In order to find possible candidate genes, we analyzed the homology between these 39 genes and 20 characterized cold-tolerance genes, LOC_Os09g12440, LOC_OsO9g12470, LOC_Os09g12520, LOC_Os09g12580 and LOC_Os09g12720 were found having a high degree of homology with COLD1, Ctb1, LTG1, OsWRKY71 and OsbZIP73(Fig. 60Table 4).

\section{Discussion}

\section{Population structure and phenotypic assessment of a natural population}

For this study, GWAS was used as a method to reveal complicated genetic variations of cold tolerance. However, population structure is an important factor affecting the results of GWAS and increases the false positive rate. In this study, a natural population consisting of 211 rice landraces (130 indica rice and 81 japonica rice) was used to assess cold tolerance in rice at the bud burst stage. Most of the rice varieties come from 15 provinces in China, with three from Japan and one from Philippines. The geographical regions spans from the north latitude $15^{\circ}$ to $48^{\circ}$ including temperate zone, tropics and subtropics. This natural population is newly constructed and has rich genetic diversity. Population structure analysis divided the natural population into three groups. Subsequently, the results of PCA and $\mathrm{NJ}$ trees support this result that low relatedness were showed from the relative kinship analysis (Fig. 2), which makes it suitable for GWAS.

We used the SSR as the indicator to evaluate the cold tolerance of natural populations. The results show that the SSR ranges from $0 \%$ to $100 \%$ (Table S2), indica rice is extremely sensitive to temperature, and its SSR was low after cold treatment. Some indica rice varieties even die in the recovery time, while japonica rice is with the characteristic of cold tolerance, and the SSR of most japonica rice is above $90 \%$. This result showed that japonica rice was more tolerance to cold than indica rice at the bud burst stage.

\section{Identification of QTLs/candidate genes controlling CTBB}

In this study, we found 12 QTLs using SSR as indicator. Among these QTLs, seven of them ( $q S S R 2-1$, qSSR3-4, qSSR3-5, qSSR3-6, qSSR3-7, qSSR4 and qSSR7) were reported for the first time, and the other five QTLs ( $q S S R 2-2$, qSSR3-1, qSSR3-2, qSSR3-3 and qSSR9) were co-located with previous studies. The physical distance of the peak SNP for $q S S R 2-2$ was located at 4.4Mb on chromosome 2. It overlapped 
with OSWRKY71 that is a transcriptional suppressor that encodes GA signaling in aleurone cells and coldtolerant [28]). qSSR3-1, qSSR3-2 and qSSR3-3 were co-located with qLVG3 [29], a QTL for low-temperature vigor of germination. In $q L V G 3$ region,, there were two characterized cold-tolerance genes (OSMYB2 and OsCIPKO3). OSMYB2 is a MYB transcription factor and plays a regulatory role in tolerance of rice to salt, cold injury and dehydration stress [30], while OSCIPKO3 is a calcineurin B-like protein-interacting protein kinases, the overexpression of OSCIPKO3 transgenic plants significantly improved the tolerance to cold stress [31]. The QTL $q S S R 9$ explained the largest phenotype variation in our study overlapping with clr9 that is a QTL associated with culm length growth rate under cold stress [32], however, the knowlwdge of candidate genes underlying $q S S R 9$ is still gaping.

The abundant SNPs dataset of our nature population through chip strategy makes it feasible to locate qSSR9 on a small genomic region. The analysis of candidate genes shows that there are 39 candidate genes undelying $q S S R 9$, among these candidate genes, five genes (LOC_Os09g12440, LOC_Os09g12470, LOC_Os09g12520, LOC_Os09g12580 and LOC_Os09g12720) might be the target genes, because these five candidated genes share the same branch of the characterized cold tolerance genes COLD1, Ctb1, LTG1, OsWRKY71 and OsbZIP73, respectively (Fig. 6). For theses characterized cold tolerance genes, COLD1 encodes a $G$ protein signal regulator and it can interact with RGA1, the a subunit of $G$ protein, to sense low temperature, activate $\mathrm{Ca}^{2+}$ channel, and enhance the activity of $\mathrm{G}$ protein GTP-enzyme to enhance cold tolerance of rice [10]; Ctb1 encodes F-box protein, which interacts with an E3 ubiquitin ligase subunit SKP1 and is involved in cold tolerance at booting stage [12]; LTG1 encodes casein kinase and regulates cold response in rice and affects auxin transport, synthesis and signal transduction, and positively regulates low temperature tolerance of rice during vegetative growth period [33]; OsbZIP73 Jap is up-regulated by low temperature and the plant hormone abscisic acid (ABA), suggesting that OsbZIP73 is involved in ABA-dependent low temperature signaling pathways [34]. However, that other genes underlying qSSR9 cannot be ruled out, such as LOC_Os09g12360, LOC_Os09g12390, LOC_Os09g12450, LOC_Os09g12615, LOC_Os09g12640 and LOC_Os09g12650. Although these genes do not share the same branches with the characterized cold tolerance genes, they are very homologous with some the characterized cold tolerance genes (Fig. 6). Further studies, such as, gene complementation analysis, are necessary to elucidate which allele is more favourable.

\section{Conclusions}

In this study, a natural population consisting of 211 rice landraces were used to assess CTBB by using GWAS under a mixed linear model, 12 QTLs were detected on chromosomes $2,3,4$, 7, and 9, five genes (LOC_Os09g12440, LOC_Os09g12470, LOC_Os09g12520, LOC_Os09g12580 and LOC_Os09g12720) might be the target genes for $q S S R 9$ after candidate gene analysis, these QTLs/genes will be conducive to further mining favorable gene resources and breeding of rice varieties.

\section{Materials And Methods}

\section{Plant material}


A natural population of 211 rice varieties was used to evaluate the cold tolerance of rice at the bud burst stage (Table S1). These varieties were mainly collected from 15 different provinces in China as well as from Philippines and Japan. The geographical position spans from the north latitude $15^{\circ}$ to $48^{\circ}$. These regions including temperate, tropical and subtropical regions. Of the 211 rice varieties, 81 were classified as japonica rice, and 130 were classified as indica rice. The rice materials were collected in accordance with local laws without any dispute of interest. The population was developed in the experimental field at Jiangxi Agricultural University in Nanchang, Jiangxi Province, and Linwang, Hainan Province for more than two generations.

\section{Cold tolerance evaluation at the bud burst stage}

The tested seeds were placed in an oven at $45^{\circ} \mathrm{C}$ for 48 hours to break seed dormancy. The seeds were disinfected with sodium hypochlorite solution and rinsed with sterile water for three times. Next, the seeds were soaked in water for about 48 hours and allowed to germinate for 24 hours. 30 seeds, $5 \mathrm{~mm}$ in coleoptile length, were selected and placed in a petri dish with two sheets of filter paper. The petri dish were placed in a growth incubator at $4^{\circ} \mathrm{C}$, and treated in darkness for 10 days. After cold treatment, the petri dishes containing the seeds were placed in an incubator at $25^{\circ} \mathrm{C}(14-\mathrm{h}$ light/10-h dark) and recovered for 5 days. the seedlings survival rate (SSR) was calculated after 5 days of recovery of growth and used as the indicator of CTBB. seedlings survival rate $(\%)=$ surviving seed $/ 30 \times 100$, All experiments were repeated for three times.

\section{GWAS Mapping of Rice CTBB QTLs/genes}

Tassel 5.0 software was used for GWAS of rice (CTBB). Principal component analysis (PCA) and kinship analysis were performed using 36,727 SNP genotype dataset. After filtering, standardized phenotype data, SNP data and principal component data were merged, and GWAS was performed with kinship data as co-variables under a mixed linear model. Plotting with R package (R_MVP). QTL regions were identified when 3 or more significant SNPs occurred within $400 \mathrm{~kb}$ interval. Candidate gene analyses of $q S S R 9$ were conducted by the RAP-DB (https://rapdb.dna.affrc.go.jp/) and NCBI (https://www.ncbi.nlm.nih.gov/). The DNA sequences of the putative genes and cold tolerance genes were downloaded from NCBI and used in MEGA-X software (https://www.megasoftware.net/).

\section{Statistical and genetic analyses}

A correlation analysis of the SSR, latitude and Indica or japonica type was carried out by SPSS Statistics 26 software.

\section{Genomic DNA extraction, sequencing and genotyping}

The CTAB method was used to extract DNA from about $100 \mathrm{mg}$ of fresh young leaves, and the quantity and quality of DNA were measured using a Denovix DS-11 spectrophotometer. In addition, purity was determined by the result of running the DNA for $1 \mathrm{~h}$ at $60 \mathrm{~V}$ in $1 \%$ agarose gel electrophoresis. The $50 \mathrm{~K}$ 
rice gene SNP microarray 'OsSNPNKs' was used for genotyping. The microarrays were evenly distributed throughout the genome, with an average distance of $<1 \mathrm{~Kb}$ between each other. Genotyping based on Affymetrix AXIOM ®2.0. The Target Prep Protocol QRC (P/N 702990) kit manual was used for DNA amplification, DNA fragmentation, microarray hybridization, DNA binding single base extension, and signal amplification. Staining and scanning were performed using a GeneTitan ${ }^{\circledR}$ multi-channel instrument [27].

\section{Abbreviations}

Seedling survival rate (SSR), quantitative trait loci (QTL), genome-wide association study (GWAS), Single nucleotide polymorphism (SNP), cold tolerance at the bud burst stage (CTBB), Principal component analysis (PCA), Neighbor-Joining (NJ).

\section{Declarations}

\section{Acknowledgments}

We thank the anonymous referees for their critical comments on this manuscript.

\section{Authors' contributions}

$C, L$ is the first author of this article, designed and performed experiments, analyzed data and wrote the manuscript. J. L, J. B participated in analysis data. T. J, B. Z, J. T, X. Z, P. W, G. W, Q. C, Y. W, Q. Z, S. H, M. $Y, T$. H, S.L, C.L participated in performing experiments. H.H and J.B. conceived and supervised the experiments. All authors reviewed the manuscript.

\section{Funding}

This research was supported by grants (2016YFD0101801) from The National Key Research and Development Program of China and Technology Department and grants (20192BCB23010, 20192ACBL20017) from Project of Science and Technology Department of Jiangxi Province and grant (202010410100, 202010410106) from National Undergraduate Training Program for Innovation and Entrepreneurship. These funding institutions provided financial support for material collection, highthroughput sequencing and data analysis in this study.

\section{Availability of data and materials}

The data supporting the conclusions of this article are included within the article and its additional files.

\section{Ethics approval and consent to participate}

Not applicable. 
Not applicable.

\section{Competing interests}

The authors declare that the research was conducted in the absence of any commercial or financial relationships that could be construed as a potential conflict of interest.

\section{Author details}

${ }^{1}$ Key Laboratory of Crop Physiology, Ecology and Genetic Breeding, Ministry of Education, Nanchang 330045, China.

${ }^{2}$ Key Laboratory of Crop Physiology, Ecology and Genetic Breeding, Jiangxi Province, Nanchang 330045, China.

${ }^{3}$ Agricultural Genomics Institute in Shenzhen, Chinese Academy of Agricultural Sciences, Shenzhen Guangdong.

${ }^{4}$ Peking University Institute of Advanced Agricultural Sciences, Weifang 261325, Shandong, China

*Corresponding author: jmbian81@126.com (Jianmin Bian); hhhua64@163.com (Haohua He)

\section{References}

1. Sthapit BR, Witcombe JR. Inheritance of tolerance to chilling stress in rice during germination and plumule greening. Crop Sci.1998; 38:660-665

2. Dai LY, Ye CR, Yu TQ, Xu FR. Studies on cold tolerance of rice, Oryza sativa L:X. Description on types of cold injury and classifications of evaluation methods on cold tolerance in rice. Southwest China $\mathrm{J}$ Agric Sci. 2002; 15(1):41 45

3. Zhang Q, Chen Q, Wang S. Rice and cold stress: methods for its evaluation and summary of cold tolerance-related quantitative trait loci. Rice. 2014; 7, 24

4. Fujino K, Sekiguchi H, Matsuda Y, Sugimoto K, Ono K, Yano M. Molecular identification of a major quantitative trait locus, qLTG3-1, controlling low-temperature germinability in rice. Proc Natl Acad Sci USA. 2008; 105(34), 12623-12628

5. Andaya VC, Tai TH. Fine mapping of the qCTS12 locus, a major QTL for seedling cold tolerance in rice. Theor Appl Genet. 2006; 113:467-475

6. Andaya VC, Tai TH. Fine mapping of the qCTS4 locus associated with seedling cold tolerance in rice (Oryza sativa L.). Mol Breed. 2007; 20:349-358

7. Koseki M, Kitazawa N, Yonebayashi S, Maehara Y, Wang ZX, Minobe Y. Identification and fine mapping of a major quantitative trait locus originating from wild rice, controlling cold tolerance at the seedling stage. Mol Genet Genomics. 2010; 284:45-54 
8. Kim SM, Suh JP, Lee CK, Lee JH, Kim YG, Jena KK. QTL mapping and development of candidate gene-derived DNA markers associated with seedling cold tolerance in rice (Oryza sativa L.). Mol Genet Genomics. 2014; 289:333-343

9. Xiao N, Huang WN, Li AH, Gao Y, Li YH, Pan CH, Ji H, Zhang XX, Dai Y, Dai ZY, Chen JM. Fine mapping of the $q L O P 2$ and $q P S R 2-1$ loci associated with chilling stress tolerance of wild rice seedlings. Theor Appl Genet. 2015; 128:173-185

10. Yun Ma, Xiaoyan Dai, Yunyuan Xu, Wei Luo. COLD1 confers chilling tolerance in rice. Cell. 2015; 160:1209-1221

11. Zhao J, Zhang S, Dong J. A novel functional gene associated with cold tolerance at the seedling stage in rice. Plant Biotechnol J. 2017;15(9):1141-1148

12. Saito K, Hayano-Saito Y, Maruyama-Funatsuki W, Sato Y, Kato A. Physical mapping and putative candidate gene identification of a quantitative trait locus $C t b 1$ for cold tolerance at the booting stage of rice. Theor Appl Genet. 2004; 109:515-522

13. Kuroki M, Saito K, Matsuba S, Yokogami N, Shimizu H, Ando I, Sato Y. A quantitative trait locus for cold tolerance at the booting stage on rice chromosome 8. Theor Appl Genet. 2007; 115:593-600

14. Zhou L, Zeng Y, Zheng W, Tang B, Yang S, Zhang H, Li J, Li Z. Fine mapping a QTL qCTB7 for cold tolerance at the booting stage on rice chromosome 7 using a near-isogenic line. Theor Appl Genet. 2010; 121:895-905

15. Shirasawa S, Endo T, Nakagomi K, Yamaguchi M, Nishio T. Delimitation of a QTL region controlling cold tolerance at booting stage of a cultivar, 'Lijiangxintuanheigu', in rice, Oryza sativa L. Theor Appl Genet. 2012; 124:937-946

16. Zhu Y, Chen K, Mi X, Chen T, Ali J, Ye G, Xu J, Li Z. Identification and fine mapping of a stably expressed QTL for cold tolerance at the booting stage using an interconnected breeding population in rice. PloS One. 2015; 10:e0145704

17. Bian JM, Jiang L, Liu LL, Wei X J. Construction of a new set of rice chromosome segment substitution lines and identification of grain weight and related traits QTLs. Breed Sci. 2010; 60(4): p. 305-313

18. Fujino K, Obara M, Shimizu T, Koyanagi KO, Ikegaya T. Genome-wide association mapping focusing on a rice population derived from rice breeding programs in a region. Breed Sci. 2015; 65:403-410

19. Ying Pan, Hong ZH. Genetic analysis of cold tolerance at the germination and booting stages in rice by association mapping. PloS One. 2015; 10:e0120590

20. Lv Y, Guo ZL, Li XK, Ye HY, Li XH, Xiong LZ. New insights into the genetic basis of natural chilling and cold shock tolerance in rice by genome-wide association analysis. Plant Cell Environ. 2016; 39:556570

21. Wang D, Liu J, Li C. Genome-wide association mapping of cold tolerance genes at the seedling stage in rice. Rice. 2016; 9:61

22. Shakiba E, Edwards JD, Jodari F. Genetic architecture of cold tolerance in rice (Oryza sativa) determined through high resolution genome-wide analysis. PloS One. 2017; 12:e0172133 
23. Sales E, Viruel J, Domingo C, Marqués L. Genome wide association analysis of cold tolerance at germination in temperate japonica rice (Oryza sativa L.) varieties. PloS One. 2017; 12(8):e0183416

24. Zhang M, Ye J, Xu Q. Genome-wide association study of cold tolerance of Chinese indica rice varieties at the bud burst stage. Plant Cell Rep. 2018; 37:529-539

25. Song J, Li J, Sun J, Hu T, Wu A,Liu S, Wang W, Ma D and Zhao M. Genome-Wide Association Mapping for Cold Tolerance in a Core Collection of Rice (Oryza sativa L.) Landraces by Using HighDensity Single Nucleotide Polymorphism Markers From Specific-Locus Amplified Fragment Sequencing. Front. Plant Sci. 2018; 9:875.

26. Thapa, Ranjita, Tabien, Rodante, Thomson, Michael, Septiningsih, Endang. Genome-Wide Association Mapping to Identify Genetic Loci for Cold Tolerance and Cold Recovery During Germination in Rice. Front Genet. 2020; 11:22

27. Rohilla M, Singh N, Mazumder A. Genome-wide association studies using $50 \mathrm{~K}$ rice genic SNP chip unveil genetic architecture for anaerobic germination of deep-water rice population of Assam, India. Mol Genet Genomics, 2020; 295, 1211-1226.

28. Kim CY, Vo K, T X, Nguyen C.D. Functional analysis of a cold-responsive rice WRKY gene, OsWRKY71 . Plant Biotechnol Rep. 2016; 10, 13-23.

29. Han LZ, Zhang YY, Qiao YL, Cao GL, Zhang SY, Kim JH, Koh HJ. Genetic and QTL analysis for lowtemperature vigor of germination in rice. Acta Genet Sin. 2006; 33:998-1006

30. Yang A, X Dai, W Zhang. A R2R3-type MYB gene, OsMYB2, is involved in salt, cold, and dehydration tolerance in rice. J Exp Bot. 2012; 63(7): p. 2541-2556.

31. Yong X, Yue H, Li X. Characterization of Stress-Responsive CIPK Genes in Rice for Stress Tolerance Improvement. Plant Physiol. 2007; 144(3): 1416-1428

32. Oh CS, Choi YH, Lee SJ, Yoon DB, Moon HP, Ahn SN. Mapping of Quantitative Trait Loci for Cold Tolerance in Weedy Rice. Breeding Sci. 2004; 54, 373-380.

33. Lu G, Wu FQ, Wu W. Rice LTG1 is involved in adaptive growth and fitness under low ambient temperature. The Plant Journal. 2014; 78(3): p. 468-480.

34. C Liu, S Ou, B Mao, W Wei. Early selection of bZIP73 facilitated adaptation of japonica rice to cold climates. Nat Commun. 2018; 9(1): p. 3302-12.

\section{Tables}


Table 1

SSR (seedling survival rate) of cold tolerance at the bud burst stage (CTBB)

\begin{tabular}{|llll|}
\hline Seed survival rate (\%) & The total number & Number of indica & Number of japonica \\
\hline $0 \leq X \leq 20$ & 101 & 99 & 2 \\
\hline $20<X \leq 40$ & 19 & 17 & 2 \\
\hline $40<X \leq 60$ & 11 & 8 & 3 \\
\hline $60<X \leq 80$ & 11 & 1 & 10 \\
\hline $80<X \leq 100$ & 69 & 5 & 64 \\
\hline
\end{tabular}

Table 2

Correlations between SSR, latitude and indica or japonica type

\begin{tabular}{|lccl|}
\hline Traits & SSR & Latitude & Indica or japonica type \\
\hline SSR & 1 & $0.714^{\star *}$ & $0.851^{\star *}$ \\
\hline Latitude & 1 & $0.910^{\star \star}$ \\
\hline Indica or japonica type & & 1 \\
\hline **Indicates significance at the $1 \%$ levels & \\
\hline
\end{tabular}


Table 3

Summary of the significant SNPs detected by GWAS and the overlapped QTLs/genes reported previously

\begin{tabular}{|c|c|c|c|c|c|c|}
\hline QTL ID & Trait & Chr. & $\begin{array}{l}\text { Peak } \\
\text { SNPs }\end{array}$ & $P$ value & $\begin{array}{l}\text { Previous } \\
\text { QTL/genes }\end{array}$ & References \\
\hline $\begin{array}{l}q S S R 2- \\
1\end{array}$ & $\begin{array}{l}\text { seedling survival } \\
\text { rate }\end{array}$ & 2 & 3379509 & $\begin{array}{l}8.99 \mathrm{E}- \\
06\end{array}$ & & \\
\hline $\begin{array}{l}q S S R 2- \\
2\end{array}$ & $\begin{array}{l}\text { seedling survival } \\
\text { rate }\end{array}$ & 2 & 4435145 & $\begin{array}{l}9.70 \mathrm{E}- \\
07\end{array}$ & OsWRKY71 & $\begin{array}{l}\text { Kim et al. } \\
(2016)\end{array}$ \\
\hline qSSR3- & $\begin{array}{l}\text { seedling survival } \\
\text { rate }\end{array}$ & 3 & 10230171 & $\begin{array}{l}3.16 \mathrm{E}- \\
06\end{array}$ & qLVG3 & $\begin{array}{l}\text { Han et al. } \\
(2006)\end{array}$ \\
\hline $\begin{array}{l}q S S R 3- \\
2\end{array}$ & $\begin{array}{l}\text { seedling survival } \\
\text { rate }\end{array}$ & 3 & 10552889 & $\begin{array}{l}1.48 \mathrm{E}- \\
06\end{array}$ & qLVG3 & $\begin{array}{l}\text { Han et al. } \\
(2006)\end{array}$ \\
\hline \multirow[t]{3}{*}{$\begin{array}{l}q S S R 3- \\
3\end{array}$} & $\begin{array}{l}\text { seedling survival } \\
\text { rate }\end{array}$ & 3 & 11316517 & $\begin{array}{l}1.64 \mathrm{E}- \\
06\end{array}$ & qLVG3 & $\begin{array}{l}\text { Han et al. } \\
(2006)\end{array}$ \\
\hline & & & & & OsMYB2 & $\begin{array}{l}\text { Yang et al. } \\
(2012)\end{array}$ \\
\hline & & & & & OsCIPKO3 & $\begin{array}{l}\text { Xiang et al. } \\
\text { (2007) }\end{array}$ \\
\hline $\begin{array}{l}q S S R 3- \\
4\end{array}$ & $\begin{array}{l}\text { seedling survival } \\
\text { rate }\end{array}$ & 3 & 13860165 & $\begin{array}{l}1.72 \mathrm{E}- \\
08\end{array}$ & & \\
\hline $\begin{array}{l}\text { qSSR3- } \\
5\end{array}$ & $\begin{array}{l}\text { seedling survival } \\
\text { rate }\end{array}$ & 3 & 15042189 & $\begin{array}{l}2.19 \mathrm{E}- \\
08\end{array}$ & & \\
\hline $\begin{array}{l}q S S R 3- \\
6\end{array}$ & $\begin{array}{l}\text { seedling survival } \\
\text { rate }\end{array}$ & 3 & 29271308 & $\begin{array}{l}4.89 \mathrm{E}- \\
05\end{array}$ & & \\
\hline qSSR3- & $\begin{array}{l}\text { seedling survival } \\
\text { rate }\end{array}$ & 3 & 33444741 & $\begin{array}{l}7.77 \mathrm{E}- \\
06\end{array}$ & & \\
\hline qSSR4 & $\begin{array}{l}\text { seedling survival } \\
\text { rate }\end{array}$ & 4 & 11487556 & $\begin{array}{l}2.46 \mathrm{E}- \\
05\end{array}$ & & \\
\hline qSSR7 & $\begin{array}{l}\text { seedling survival } \\
\text { rate }\end{array}$ & 7 & 29111646 & $\begin{array}{l}1.77 \mathrm{E}- \\
05\end{array}$ & & \\
\hline qSSR9 & $\begin{array}{l}\text { seedling survival } \\
\text { rate }\end{array}$ & 9 & 7106185 & $\begin{array}{l}4.07 \mathrm{E}- \\
09\end{array}$ & $c / r 9$ & Oh et al.(2004) \\
\hline
\end{tabular}


Table 4

Candidate genes in the $q S S R 9$ region

\begin{tabular}{|clll|}
\hline QTL & Putative genes & Putative protein functions & $\begin{array}{c}\text { Reference } \\
\text { genes }\end{array}$ \\
\hline qSSR9 & LOC_Os09g12440 & $\begin{array}{l}\text { retrotransposon protein, putative, unclassified, } \\
\text { expressed }\end{array}$ & COLD1 \\
\hline LOC_Os09g12470 & hypothetical protein & CTB1 \\
\hline LOC_0s09g12520 & hypothetical protein & LTG1 \\
\hline LOC_Os09g12580 & expressed protein & OsWRKY71 \\
\hline LOC_Os09g12720 & $\begin{array}{l}\text { zinc finger, C3HC4 type domain containing protein, } \\
\text { expressed }\end{array}$ & OsbZIP73 \\
\hline
\end{tabular}

\section{Figures}

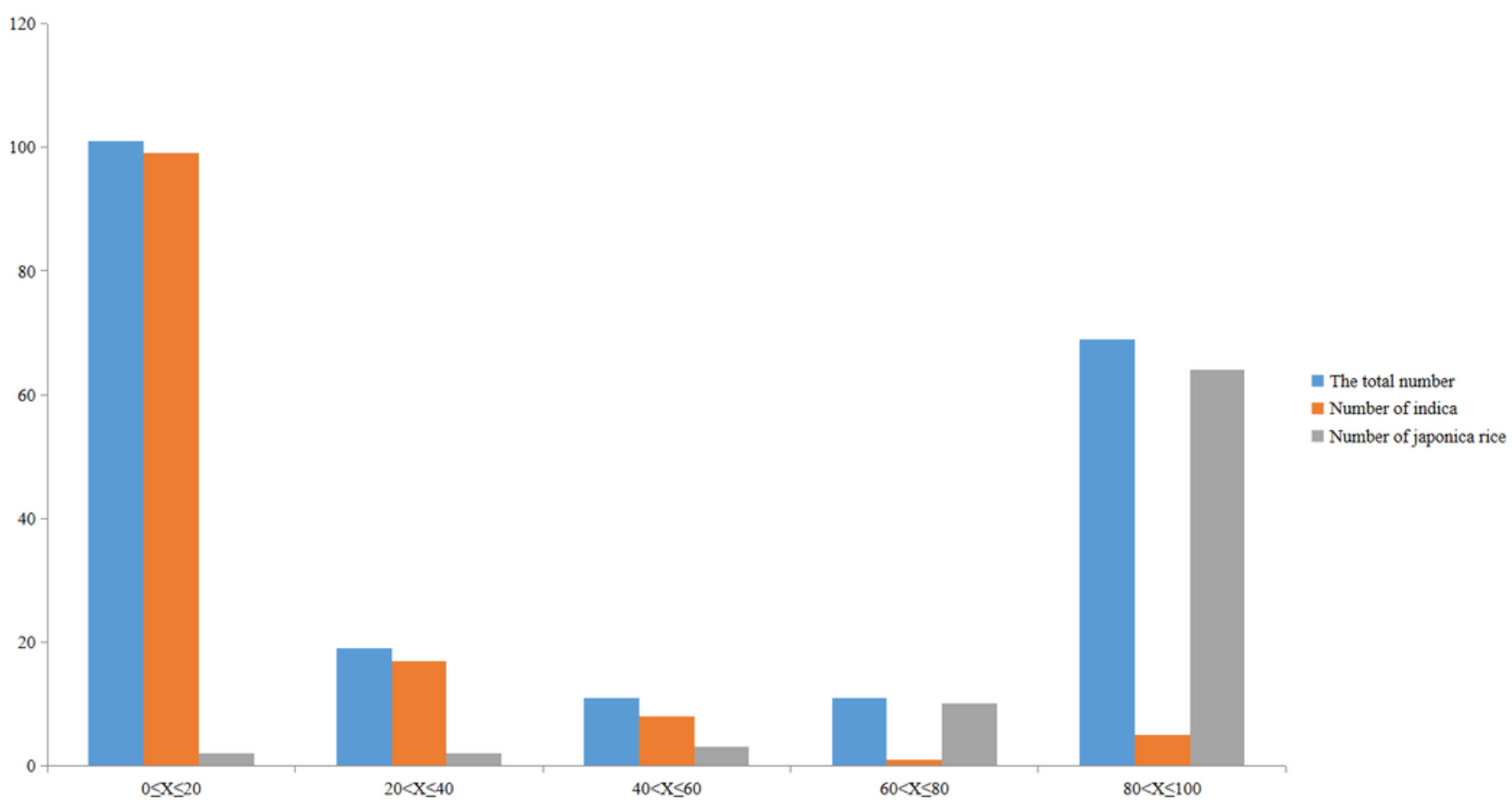

Figure 1

SSR of histogram. The blue area represents the total number of rice, the orange area represents indica rice, and the gray area represents japonica rice, and the horizontal axis represents the cold tolerance level. 
A

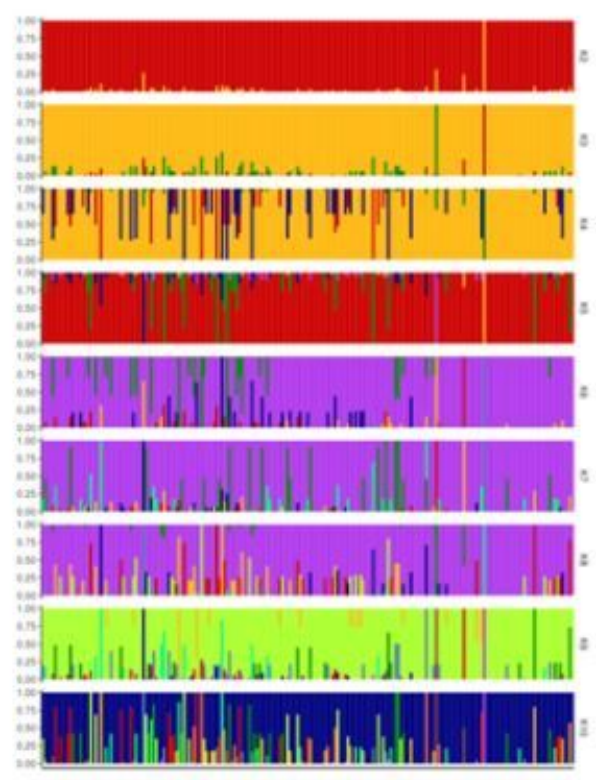

$\mathrm{C}$

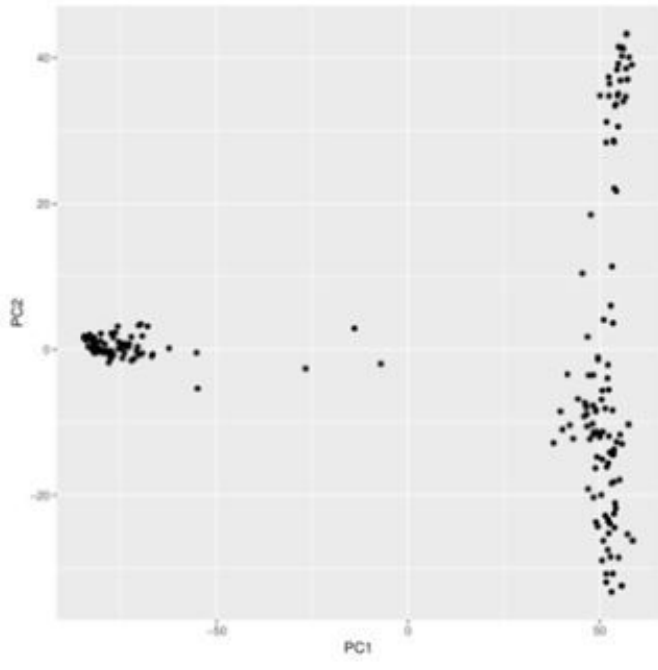

B

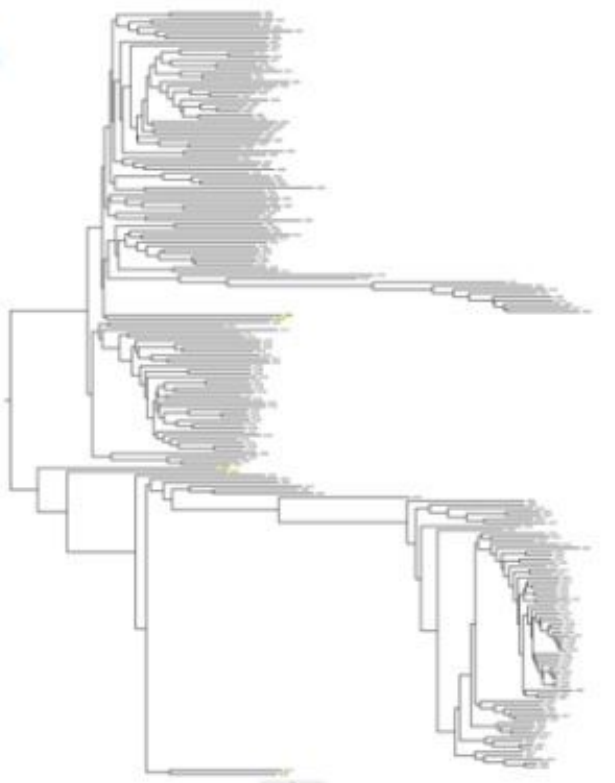

$\mathrm{D}$

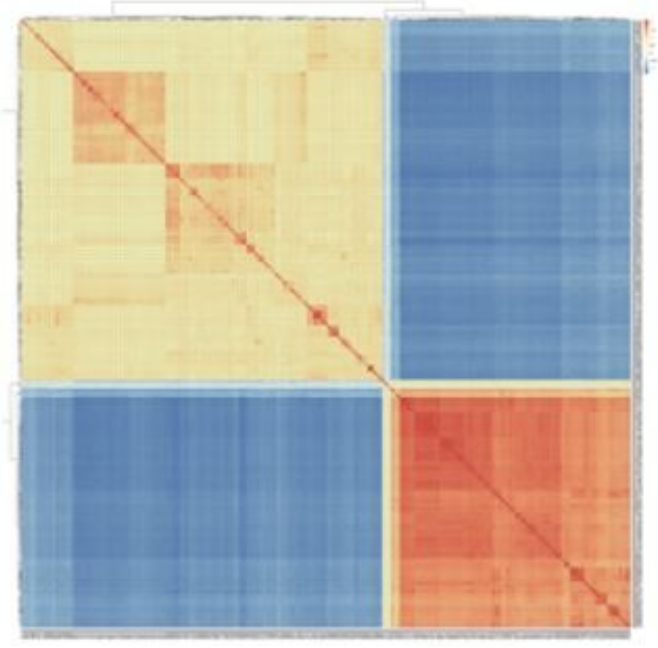

\section{Figure 2}

Population structure of 211 landraces A. Subgroups $(K=3)$ inferred using structure software, yellow, red, and green represent subgroup II, II, and III, respectively; B. NJ tree based on Nei's genetic distances; C. PCA of 211 rice varieties; D. Pairwise relative kinship analysis of rice panel. 


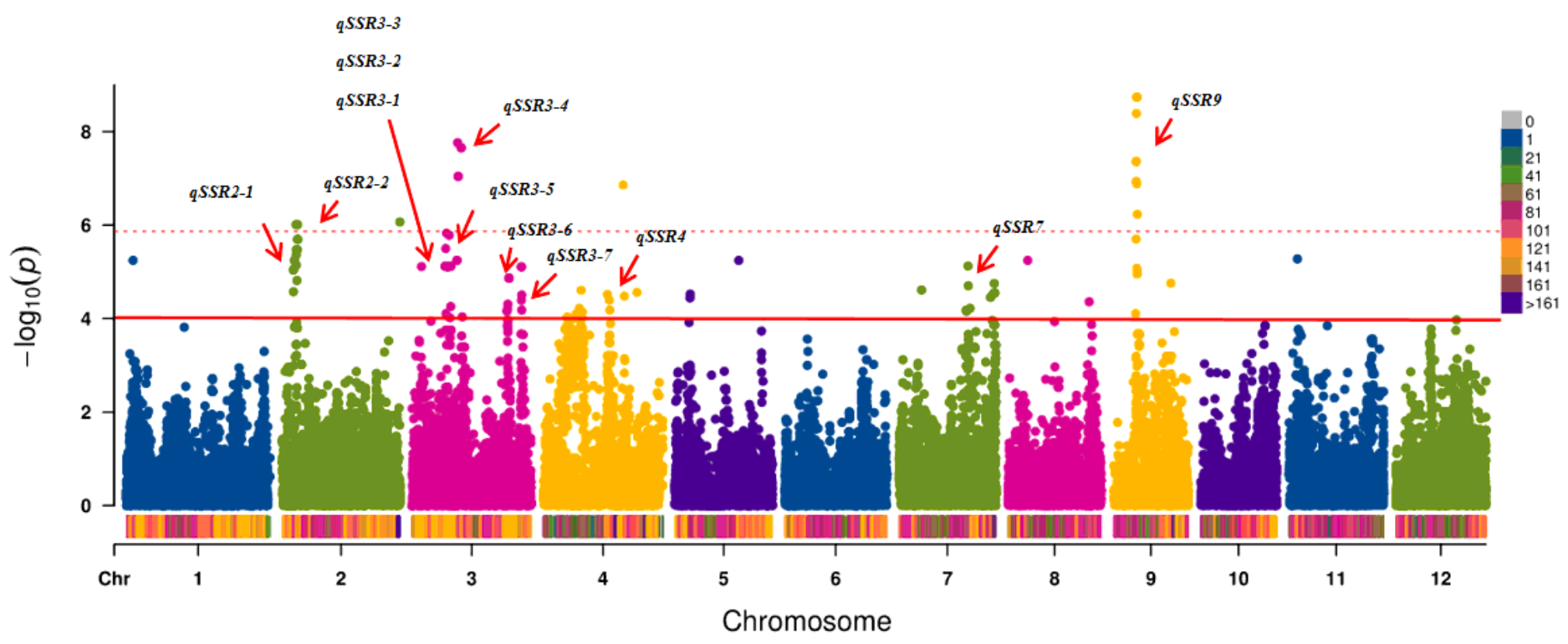

Figure 3

Manhattan plots of GWAS for SSR. The dotted line represents the Bonferoni correction and the solid line represents $\mathrm{P}=0.0001$ 


\section{QQplot of survival.MLM_MVP}

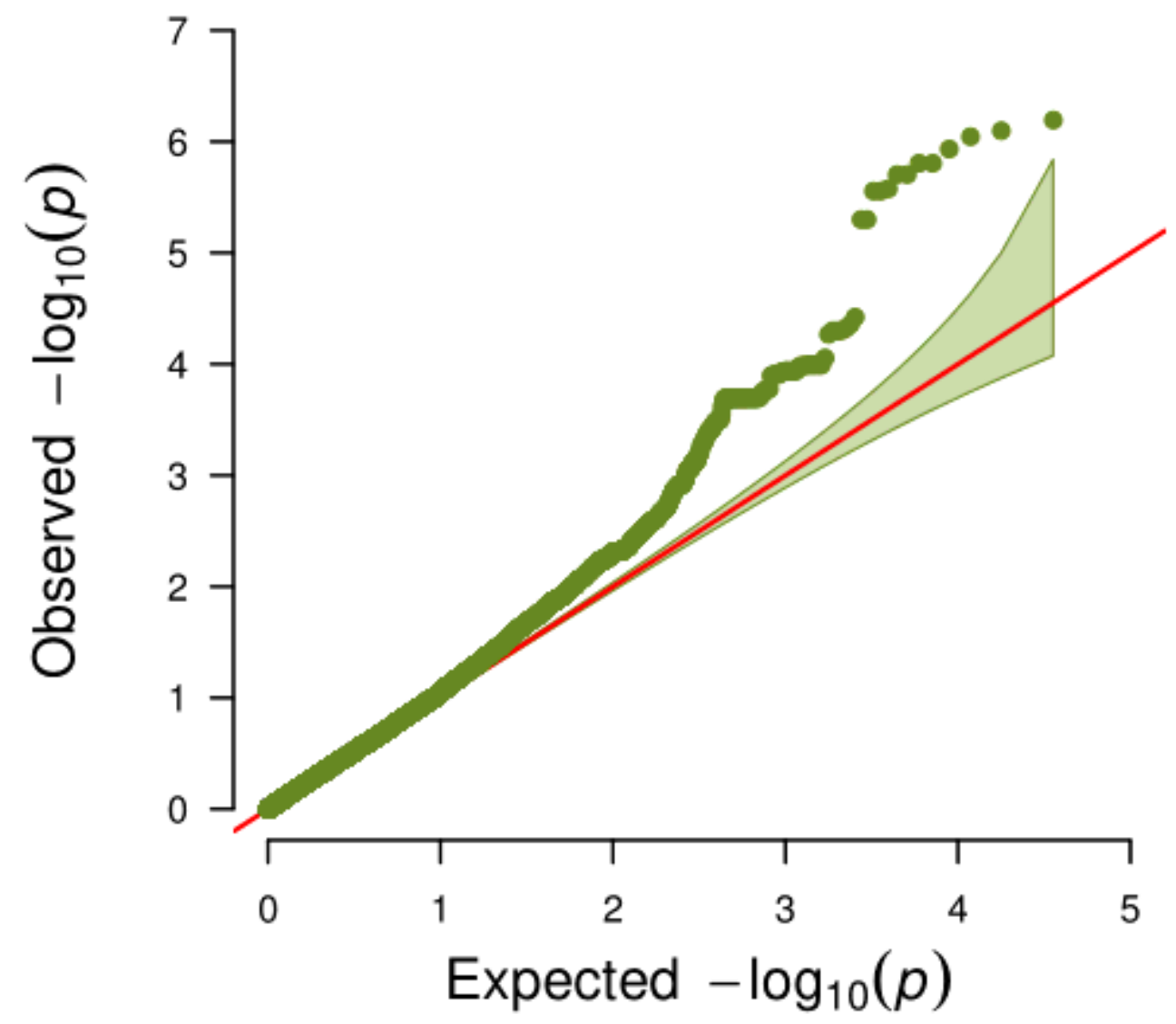

Figure 4

Quantile-quantile (Q-Q) plot of GWAS for SSR 


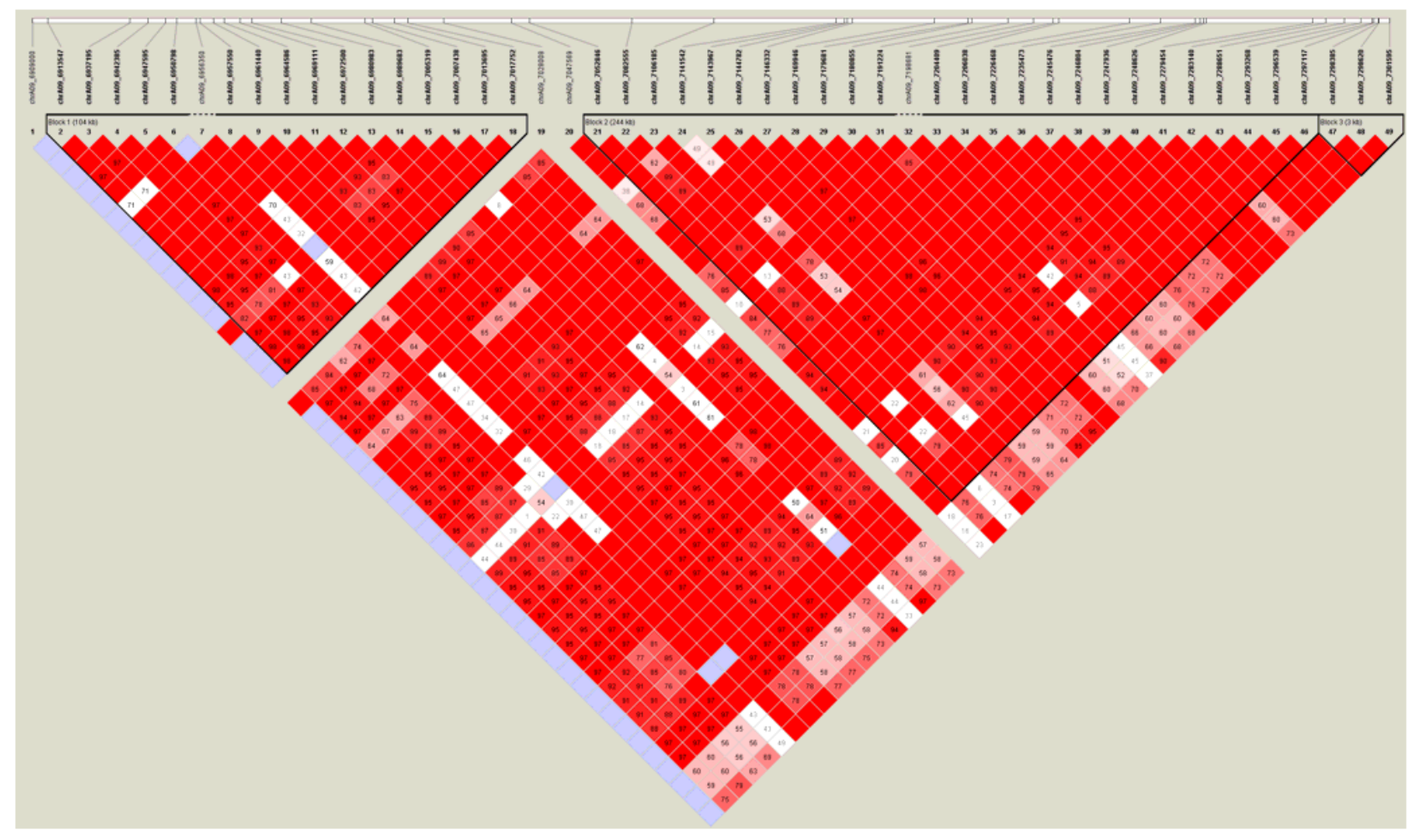

Figure 5

LD heatmap around peak on chromosome 9 


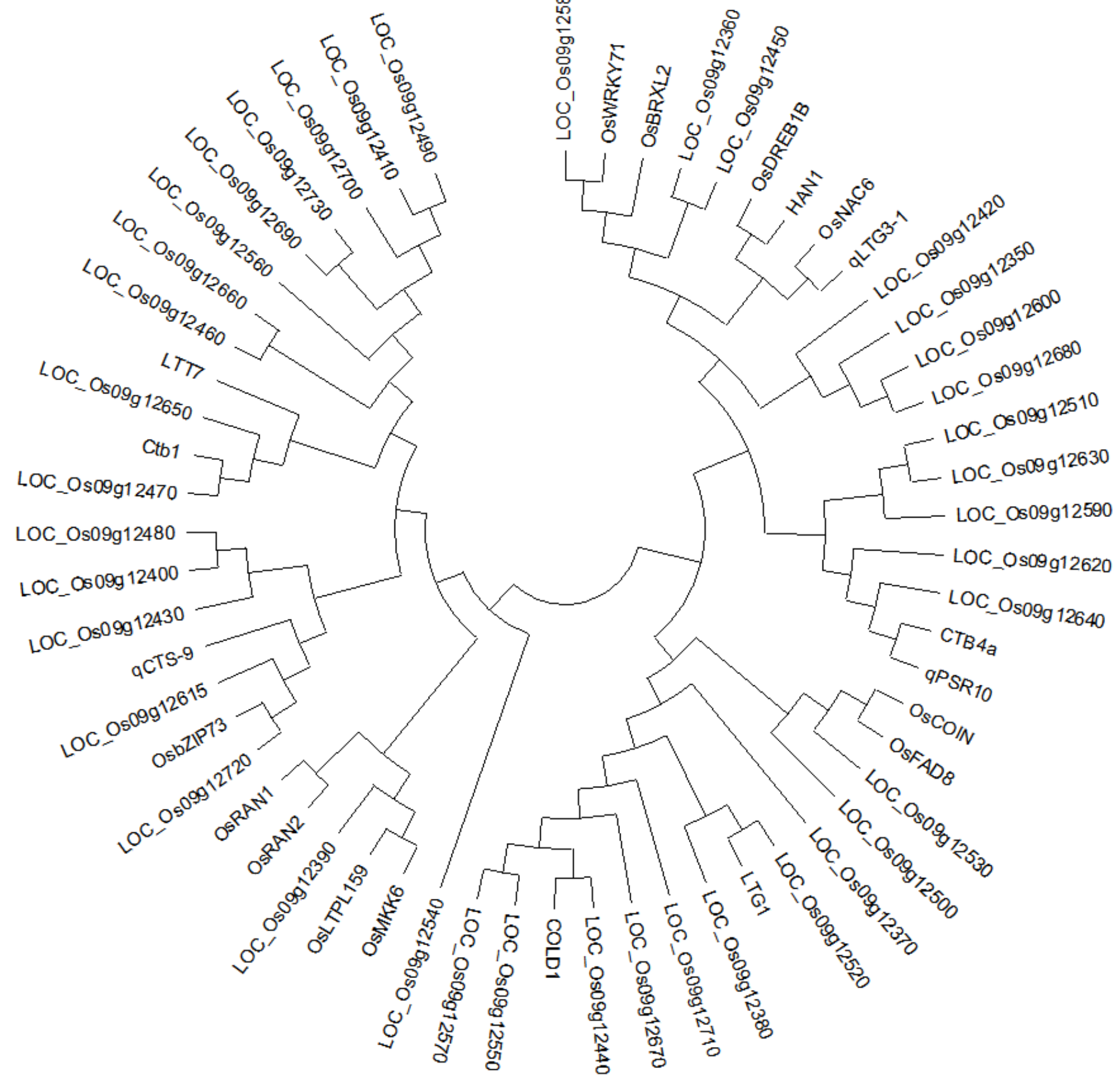

Figure 6

Homology analysis among 59 genes(39 putative genes and 20 reference genes)

\section{Supplementary Files}

This is a list of supplementary files associated with this preprint. Click to download. 
- Tables1.xls

- TableS2.xls

- Tables3.xls 